\title{
Structure of the incommensurate phase of the quantum magnet TiOCl
}

\author{
Andreas Schönleber and Sander van Smaalen* \\ Laboratory of Crystallography, University of Bayreuth, 95440 Bayreuth, Germany \\ Lukáš Palatinus \\ Laboratory of Crystallography, EPF Lausanne, 1015 Lausanne, Switzerland
}

(Received 8 March 2006; revised manuscript received 3 May 2006; published 7 June 2006)

\begin{abstract}
The incommensurately modulated structure is reported for the low-dimensional spin-1/2 quantum magnet TiOCl. On the basis of an analysis of the structural distortions in the spin-Peierls state (stable below $T_{c 1}$ $=67 \mathrm{~K}$ ) and in the incommensurate phase (stable between $T_{c 1}$ and $T_{c 2}=90 \mathrm{~K}$ ), it is argued that both the incommensurability and the twofold superstructure at low temperatures are the result of frustration between a spin-Peierls pairing on chains of $\mathrm{Ti}$ atoms as driving force and elastic coupling between neighboring chains mediated by the oxygen atoms.
\end{abstract}

DOI: 10.1103/PhysRevB.73.214410

PACS number(s): 75.30.Et, 61.50.Ks, 61.66.Fn, 75.30.Kz

\section{INTRODUCTION}

Low-dimensional $S=1 / 2$ quantum spin systems exhibit magnetic order in a variety of ground states, that may involve charge, spin, orbital, and lattice degrees of freedom. One of the interesting features is the frustration between competing interactions favoring different ground states, that are at the origin of a possible dimensionality crossover between low-temperature and high-temperature regimes. TiOCl and $\mathrm{TiOBr}$ are isostructural, paramagnetic compounds with layered structures (Fig. 1). ${ }^{1}$ Magnetic interactions between $S=1 / 2 \mathrm{Ti}^{3+}$ ions may be present as superexchange interactions on chains Ti-O-Ti parallel to $\mathbf{a}$, as diagonal interactions along $\frac{1}{2} \mathbf{a} \pm \frac{1}{2} \mathbf{b} \pm \frac{1}{4} \mathbf{c}$ on the two-dimensional layers, or as direct exchange on chains of $\mathrm{Ti}$ atoms parallel to $\mathbf{b}$. The latter type of interactions has been shown to dominate the behaviors at low temperatures. ${ }^{2,3}$ They are responsible for the formation of a spin-Peierls state below $T_{c 1}=67 \mathrm{~K}(\mathrm{TiOCl})$ or $T_{c 1}^{\prime}$ $=27 \mathrm{~K}(\mathrm{TiOBr}){ }^{2,4-6}$

Recently, we have found that $\mathrm{TiOBr}$ possesses an incommensurately modulated crystal structure between $T_{c 1}^{\prime}$ and $T_{c 2}^{\prime}$ $=47 \mathrm{~K} .{ }^{7}$ Rückamp et al. ${ }^{8}$ have argued that the existence of an incommensurate phase at intermediate temperatures is the result of frustration of interchain interactions in $\mathrm{TiOCl}$ and $\mathrm{TiOBr}$. The existence of an incommensurately modulated structure provides an explanation for anomalies that have been found in the temperature dependencies of the magnetic susceptibilities and other physical properties. ${ }^{2,3,9,10}$

In the present contribution we report the incommensurately modulated structure of $\mathrm{TiOCl}$, as it exists between $T_{c 1}=67 \mathrm{~K}$ and $T_{c 2}=90 \mathrm{~K}$. TiOCl is found to be isostructural to $\mathrm{TiOBr}$ in all phases. The crystal structure is analyzed in view of the mechanism of competing interactions that are responsible for the intricate phase diagrams of these compounds.

\section{EXPERIMENT}

Single crystals of $\mathrm{TiOCl}$ have been grown by gas transport from $\mathrm{TiCl}_{3}$ and $\mathrm{TiO}_{2}$, resulting in yellowbrown crystals with a plate-like habit. ${ }^{1}$ A single crystal of dimensions of about $0.2 \times 0.18 \times 0.01 \mathrm{~mm}^{3}$ has been glued to a carbon fiber that was attached to a closed-cycle helium cryostat mounted on a four-circle Huber diffractometer. X-ray diffraction has been measured at beamline D3 of Hasylab at DESY (Hamburg, Germany), employing monochromatic synchrotron radiation of wavelength $0.5000(1) \AA$ and a point detector. Diffraction at room temperature confirmed the $\mathrm{FeOCl}$ structure type. ${ }^{1}$

The temperature dependence of the first component of the modulation wave vector $\mathbf{q}=\left(q_{1}, \frac{1}{2}+\delta, 0\right)$ has been determined from $q$ scans along $\mathbf{a}^{*}$ centered on $(-2,-1.5,-1)$. Below $T_{c 1}=67 \mathrm{~K}$ a single peak has been observed at $(-2,-1.5$, $-1)$ that indicates a twofold superstructure with $q_{1}=0$ and $\delta=0$ at these temperatures. ${ }^{4}$ For $T_{c 1}<T<T_{c 2}$ two peaks appear at positions $\left(-2 \pm q_{1},-1.5,-1\right)$ in each $q$ scan [Fig. 2(a)]. Values of $q_{1}$ have been determined from the distances

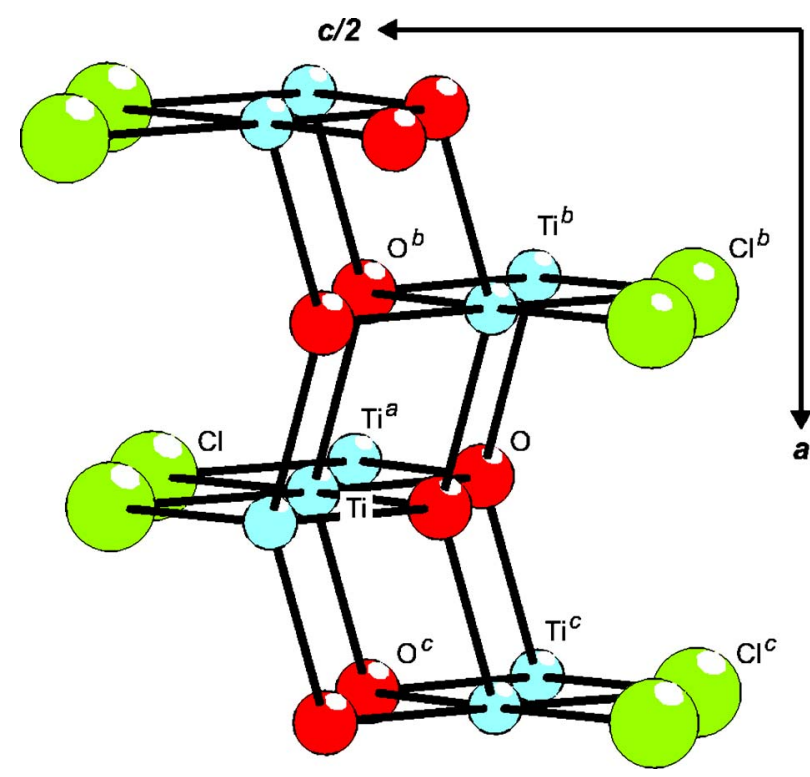

FIG. 1. (Color online) Perspective view of one layer of the crystal structure of TiOCl. Atomic labels and symmetry codes correspond to those given in Table II. Blue for Ti, red for $\mathrm{O}$, and green for $\mathrm{Cl}$. 

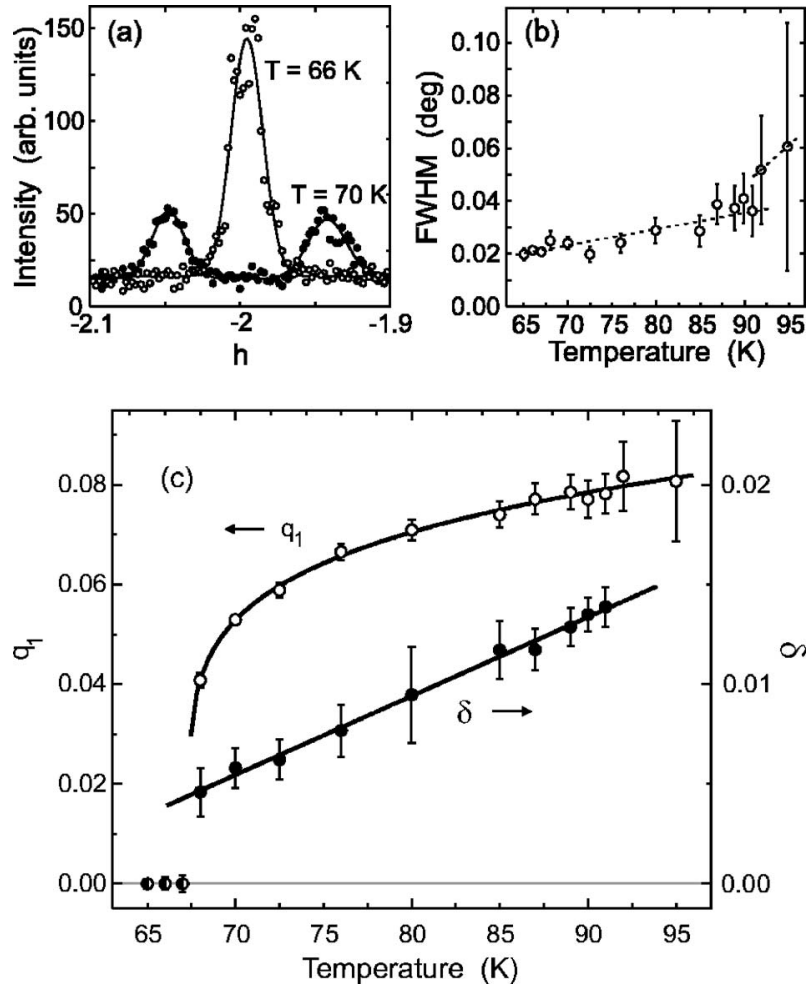

FIG. 2. (a) $q$ scans along $\mathbf{a}^{*}$ centered on $(-2,-1.5,-1)$, below and above the lock-in transition at $T_{c 1}$. Lines represent Gauss functions fitted to the data. (b) Temperature dependence of the full width at half maximum (FWHM) of the diffraction maxima, as they have been derived from the fitted Gauss functions. Dashed lines are a guide for the eye. (c) Temperature dependence of the modulation wave vector $\mathbf{q}=\left(q_{1}, \frac{1}{2}+\delta, 0\right)$. Lines represent phenomenological fits to the data: $q_{1}=q_{1}^{0}\left(T-T_{c 1}\right)^{\gamma}$ with $q_{1}^{0}=0.044(1), \gamma=0.18(1)$, and $T_{c 1}=67.4(2) \mathrm{K} ; \delta=-0.0222(8)+0.00040(1) T$. Error bars are indicated.

between these two incommensurate reflections [Fig. 2(c)]. Values of $\delta$ could not be determined from $q$ scans along $\mathbf{b}^{*}$ centered on $\left(h \pm q_{1}, k+\frac{1}{2}, l\right)$, because each incommensurate satellite appears as a single broad peak in these scans due to the relatively large mosaic spread of the crystals. Instead, $2 \theta$ scans with narrow detector slits have been performed at the positions $\left(-2-q_{1},-3.5,-1\right)$. They reveal double peaks that correspond to the different scattering angles of the reflections $\left(-2-q_{1},-3.5+\delta,-1\right)$ and $\left(-2-q_{1},-3.5-\delta,-1\right)$. Values of $\delta$ have been determined from these splittings, and $\delta$ has been found to depend linearly on temperature [Fig. 2(c)].

Intensities of the incommensurate satellite reflections decrease on increasing temperature. They do not disappear at $T_{c 2}$, but weak maxima are also found in the $q$ scans along $\mathbf{a}^{*}$ at temperatures of 92 and $95 \mathrm{~K}$, while at still higher temperatures maxima could not be found around the $(-2,-3.5,-1)$ position. Above $T_{c 2}$ the reflection maxima are broader than below $T_{c 2}$ [Fig. 2(b)], indicating that they are due to pretransitional fluctuations above a second-order phase transition at $T_{c 2}=90(1) \mathrm{K}$. Strong incommensurate fluctuations above $T_{c 2}$ are in agreement with anomalies in the temperature dependencies of NMR and the magnetic susceptibility, that are present between $T_{c 2}$ and the temperature $T^{*}=135 \mathrm{~K} \cdot{ }^{2,3,9,10}$
The persistence of the normal phase down to $T_{c 2}$, the presence of an incommensurately modulated structure between $T_{c 2}$ and $T_{c 1}$, and the presence of a twofold superstructure below $T_{c 1}$ are in complete accordance with the phase diagram of $\mathrm{TiOBr}^{7,6}$ These results are also in agreement with similar results on the incommensurate phase of $\mathrm{TiOCl}$ as they are reported by Krimmel et al. ${ }^{11}$

At $T=72 \mathrm{~K}$, the integrated intensities of the Bragg reflections up to $[\sin (\theta) / \lambda]_{\max }=0.785 \AA^{-1}$ have been measured by $\omega$ scans. Satellite reflections $\left(q_{1}, \frac{1}{2} \pm \delta, 0\right)$ as well as $\left(-q_{1}, \frac{1}{2} \pm \delta, 0\right)$ are not resolved, while these two groups of reflections are well separated. As a consequence, each measured satellite intensity corresponds to the superposition of two reflections at $\left(h+q_{1}, k+\frac{1}{2} \pm \delta, l\right)$, or at $\left(h-q_{1}, k+\frac{1}{2} \pm \delta, l\right)$. Data processing, following standard procedures, resulted in a set of observed structure factors that have been used in structure refinements.

Structure refinements have been performed within the superspace approach, ${ }^{12,13}$ employing the computer program JANA2000. ${ }^{14}$ The analysis has closely followed the structural analysis of $\mathrm{TiOBr}^{7}$ In particular, the observed diffraction pattern can be interpreted as due to a crystal with a twodimensional modulation and the orthorhombic superspace group $\operatorname{Pmmn}\left(q_{1} q_{2} 0\right)\left(-q_{1} q_{2} 0\right) 000000$, or as due to a crystal with a one-dimensional modulation and the monoclinic superspace group $P 2 / n\left(q_{1} q_{2} 0\right) 00$. In the latter case, the orthorhombic appearance of the diffraction requires the crystal to be twinned with a volume ratio 1:1 of the two twin domains. A good fit to the diffraction data has been obtained for both models, ${ }^{17}$ in accordance with the analysis of $\mathrm{TiOBr}^{7}{ }^{7} \mathrm{How}-$ ever, recent electron diffraction experiments have indicated that $\mathrm{TiOBr}$ is twinned within its incommensurate phase, thus pointing towards monoclinic symmetry for the structure of that compound..$^{15}$ Assuming $\mathrm{TiOBr}$ and $\mathrm{TiOCl}$ to be similar in this respect too, the true symmetry of the incommensurate phase of TiOCl must be monoclinic. Therefore, the present analysis is confined to the monoclinic structure model of TiOCl. The modulation is described by a single modulation wave vector $\mathbf{q}=(0.0589,0.5066,0)$, and a single-harmonic modulation function for each atom $\mathrm{Ti}, \mathrm{O}$, and $\mathrm{Cl}^{7}$

$$
\mathbf{u}\left(\bar{x}_{4}\right)=\left[u_{x}^{1} \sin \left(2 \pi \bar{x}_{4}\right), u_{y}^{1} \sin \left(2 \pi \bar{x}_{4}\right), u_{z}^{1} \cos \left(2 \pi \bar{x}_{4}\right)\right],
$$

where $\bar{x}_{4}=t+\mathbf{q} \cdot \overline{\mathbf{x}}$ and $t$ is the phase of the modulation. ${ }^{13}$ The structure model is summarized in Table I.

\section{DISCUSSION}

Modulation wave vectors in the incommensurate states have components of similar magnitudes for $\mathrm{TiOCl}$ and TiOBr [Fig. 2(c)], ${ }^{7}$ and atomic displacements form similar patterns in both compounds (Table I and Ref. 7). Together these features show equivalent behavior of $\mathrm{TiOCl}$ and $\mathrm{TiOBr}$ in all three phases between $10 \mathrm{~K}$ and room temperature. ${ }^{4,6,7}$ Displacements of the atoms in $\mathrm{TiOCl}$ are larger than the displacements in $\mathrm{TiOBr}$, in agreement with the higher transition temperatures in $\mathrm{TiOCl}$. Assuming a pairing of $\mathrm{Ti}$ atoms along b to be the driving force for the distortions, an approximate scaling is found of $u_{y}[\mathrm{Ti}-\mathrm{TiOCl}] / u_{y}[\mathrm{Ti}-\mathrm{TiOBr}]$ $\approx\left(T_{c 2} / T_{c 2}^{\prime}\right)^{1 / 2}$. 
TABLE I. Structural parameters for the three independent atoms in the incommensurate structure of TiOCl at $T=72 \mathrm{~K}$. Fractional coordinates of the basic structure $\left(x^{0}, y^{0}\right.$, and $\left.z^{0}\right)$ refer to the lattice parameters $a=3.779$ (6), $b=3.341$ (5), and $c=8.017$ (16) $\AA$. Modulation parameters $u_{x}^{1}, u_{y}^{1}$, and $u_{z}^{1}$ are given in $\AA$ [Eq. (1)]. Equivalent isotropic atomic displacement parameters $U_{i s o}^{u p}$ are given in $\AA^{2}$. Standard uncertainties are in parentheses.

\begin{tabular}{cccccccc}
\hline \hline Atom & $x^{0}$ & $y^{0}$ & $z^{0}$ & $u_{x}^{1}$ & $u_{y}^{1}$ & $u_{z}^{1}$ & $U_{i s o}^{e q}$ \\
\hline $\mathrm{Ti}$ & 0 & 0.5 & $0.11976(8)$ & $-0.0081(18)$ & $0.0490(14)$ & $-0.0141(26)$ & $0.0087(2)$ \\
$\mathrm{O}$ & 0 & 0 & $-0.05626(32)$ & $-0.0107(83)$ & $0.0351(63)$ & $0.0238(65)$ & $0.0103(8)$ \\
$\mathrm{Cl}$ & 0 & 0 & $0.33369(12)$ & $0.0009(41)$ & $-0.0133(18)$ & $-0.0592(25)$ & $0.0106(3)$ \\
\hline \hline
\end{tabular}

The twofold superstructures at low temperatures can be understood from a spin-Peierls pairing on chains of Ti atoms along $\mathbf{b},{ }^{2,4}$ together with elastic coupling between the atoms. A hypothetical structure of one ribbon would have longitudinal displacements of Ti along $\mathbf{b}$ combined with displacements of $\mathrm{O}$ and $\mathrm{Cl}$ or $\mathrm{Br}$ along c. However, this kind of displacements would lead to an unfavorable variation of the lengths of Ti-O bonds between neighboring ribbons. This is resolved by the introduction of a component along $\mathbf{c}$ for the displacement of $\mathrm{Ti}$, and of a component along $\mathbf{b}$ for the displacement of $\mathrm{O}$, such that $\mathrm{Ti}$ and $\mathrm{O}$ displacements are almost parallel (Fig. 3), and all Ti-O bonds between ribbons $\frac{1}{2} \mathbf{a}$ apart are equal to each other., ${ }^{4,6}$ Displacements of $\mathrm{Cl}$ and $\mathrm{Br}$ just follow the displacements of $\mathrm{Ti}$, as it is evidenced by equal distances between these atoms. ${ }^{4}$ Accordingly, the observed atomic displacements are the result of frustration between spin-Peierls deformation on single chains and elastic coupling between ribbons.

With frustration between intrachain and interchain coupling already present in the spin-Peierls state, the question emerges about the interactions responsible for the incommensurability of the structure at intermediate temperatures. First it is noticed, that the amplitudes of the incommensurate modulation at $72 \mathrm{~K}$ are of similar sizes as the displacements

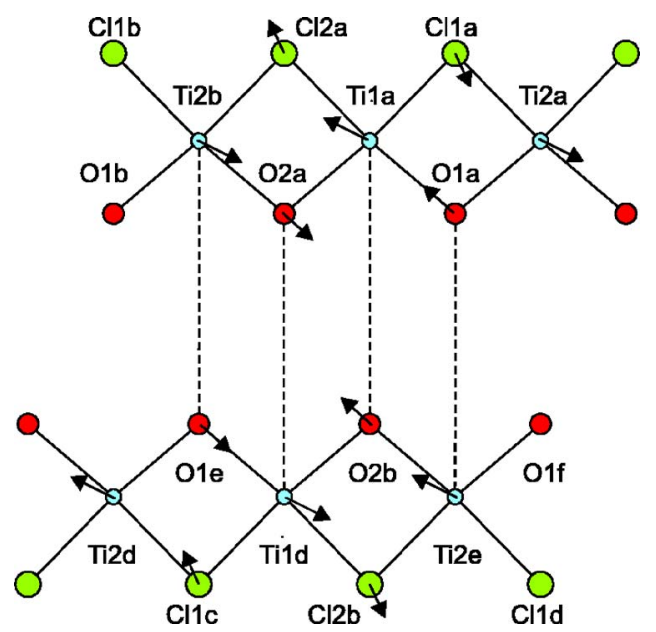

FIG. 3. (Color online) Neighboring ribbons of the structure of TiOCl, $\frac{1}{2} \mathbf{a}$ apart (compare to Fig. 1). Dashed lines represent bonds between atoms in neighboring ribbons. Arrows indicate displacements in the twofold spin-Peierls state $(20 \times$ their true values). Atomic labels and symmetry codes correspond to those given in Ref. 4. Blue for $\mathrm{Ti}$, red for $\mathrm{O}$, and green for $\mathrm{Cl}$. in the twofold superstructure at $10 \mathrm{~K}$. More precisely, minimum and maximum values of bond distances and bond angles in the incommensurate phase (Table II) are equal to the two different values of corresponding interactions in the twofold superstructure as they have been reported in Ref. 4. Thus, the incommensurate phase appears to be an incommensurate analogue of the twofold superstructure in virtually all aspects.

Ti-O-Ti bond angles and Ti-Ti distances do not show variations with the phase of the modulation that could indicate magnetic interactions other than the spin-Peierls coupling along b (Table II; Ref. 4). It follows that frustration between spin-Peierls dimerization and elastic interchain coupling is responsible for the incommensurability of the modulation as well as for the twofold superstructure. This situation is similar to that of the $A_{2} B X_{4}$ class of compounds, where frustration of elastic interactions between atoms leads to an incommensurate structure at intermediate temperatures, that locks in into a commensurate superstructure at low temperatures without entirely resolving the frustration. ${ }^{16}$ Frustration of interactions can alternatively be resolved by the formation

TABLE II. Interatomic distances $(\AA)$ and bond angles (deg). Given are the average values (Ave), the minimum and maximum values, as well as the difference $(\Delta)$ between maximum and minimum values, for variation of the phase $t$ of the modulation. Standard uncertainties are in parentheses.

\begin{tabular}{|c|c|c|c|c|}
\hline Atoms & Ave & Min & Max & $\Delta$ \\
\hline $\mathrm{Ti}^{-} \mathrm{Ti}^{\mathrm{a}}$ & $3.3417(10)$ & $3.243(2)$ & $3.438(2)$ & 0.195 \\
\hline $\mathrm{Ti}-\mathrm{Ti}^{\mathrm{b}}$ & $3.1702(19)$ & $3.1494(15)$ & $3.1916(15)$ & 0.0422 \\
\hline $\mathrm{Ti}^{\mathrm{T}} \mathrm{Ti}^{\mathrm{c}}$ & $3.1697(19)$ & $3.1435(16)$ & $3.1970(16)$ & 0.0535 \\
\hline $\mathrm{Ti}-\mathrm{Cl}$ & $2.395(2)$ & $2.387(2)$ & $2.404(2)$ & 0.017 \\
\hline Ti-O & $2.187(5)$ & $2.159(4)$ & $2.216(4)$ & 0.057 \\
\hline $\mathrm{Ti}^{\mathrm{i}} \mathrm{O}^{\mathrm{b}}$ & $1.957(5)$ & $1.952(8)$ & $1.962(8)$ & 0.010 \\
\hline Ti-Cl-Ti ${ }^{\mathrm{a}}$ & $88.53(7)$ & $84.85(9)$ & $92.15(10)$ & 7.3 \\
\hline Ti-O-Ti ${ }^{\mathrm{a}}$ & 99.62(19) & $97.0(3)$ & $102.2(3)$ & 5.2 \\
\hline $\mathrm{Ti}-\mathrm{O}-\mathrm{Ti}^{\mathrm{b}}$ & 99.67(19) & $99.5(2)$ & $99.9(2)$ & 0.4 \\
\hline $\mathrm{Ti}-\mathrm{O}-\mathrm{Ti}^{\mathrm{c}}$ & 99.66(19) & $99.2(3)$ & $100.1(3)$ & 0.9 \\
\hline $\mathrm{Ti}^{\mathrm{b}}-\mathrm{O}-\mathrm{Ti}^{\mathrm{c}}$ & $149.8(3)$ & $149.3(4)$ & $150.4(4)$ & 1.1 \\
\hline
\end{tabular}

${ }^{\mathrm{a}} x,-1+y, z$.

b $-1 / 2-x, 1 / 2-y,-z$.

${ }^{\mathrm{c}} 1 / 2-x, 1 / 2-y,-z$. 
of commensurate and incommensurate superstructures, and it thus explains the occurrence of both types of modulation. But of course, the principle of frustration by itself cannot predict the specific components of the modulations wave vectors or their temperature dependencies. For this, the free energy for the different structures need to be considered, which is beyond the scope of the present study.

\section{CONCLUSION}

By consideration of the full complexity of the crystal structures, including Ti-O bonding interactions, we have shown that frustrated interchain interactions are already present in the spin-Peierls state. They do not require a component of the displacements along $\mathbf{a}$-as it was proposed in Ref. 8 on the basis of a model involving Ti atoms only. Indeed, the structural analysis shows very small nonzero am- plitudes along a (Table I), that can hardly act as driving force for the incommensurability. The present analysis suggests that interchain interactions are of elastic origin, and that the occurrence of commensurate and incommensurate superstructures does not require magnetic interactions other than the spin-Peierls interaction. This model is in agreement with experiments and band structure calculations that have shown that the valence electron of $\mathrm{Ti}^{3+}$ occupies the $d_{x y}$ orbital at all temperatures. ${ }^{3}$

\section{ACKNOWLEDGMENTS}

Single crystals were grown by A. Suttner. We thank W. Morgenroth for assistance with the synchrotron experiment at beamline D3 of Hasylab at DESY in Hamburg, Germany. Financial support by the German Science Foundation (DFG) is gratefully acknowledged.
*Electronic address: smash@uni-bayreuth.de

†Permanent address: Institute of Physics, Czech Academy of Sciences, 16253 Praha, Czechia.

${ }^{1}$ H. Schäfer, F. Wartenpfuhl, and E. Weise, Z. Anorg. Allg. Chem. 295, 268 (1958).

${ }^{2}$ A. Seidel, C. A. Marianetti, F. C. Chou, G. Ceder, and P. A. Lee, Phys. Rev. B 67, 020405(R) (2003).

${ }^{3}$ M. Hoinkis, M. Sing, J. Schäfer, M. Klemm, S. Horn, H. Benthien, E. Jeckelmann, T. Saha-Dasgupta, L. Pisani, R. Valenti, and R. Claessen, Phys. Rev. B 72, 125127 (2005).

${ }^{4}$ M. Shaz, S. van Smaalen, L. Palatinus, M. Hoinkis, M. Klemm, S. Horn, and R. Claessen, Phys. Rev. B 71, 100405(R) (2005).

${ }^{5}$ T. Sasaki, M. Mizumaki, K. Kato, Y. Watabe, Y. Nishihata, M. Takata, and J. Akimitsu, cond-mat/0501691 (unpublished).

${ }^{6}$ L. Palatinus, A. Schönleber, and S. van Smaalen, Acta Crystallogr., Sect. C: Cryst. Struct. Commun. 61, i48 (2005).

${ }^{7}$ S. van Smaalen, L. Palatinus, and A. Schonleber, Phys. Rev. B 72, 020105(R) (2005).

${ }^{8}$ R. Rückamp, J. Baier, M. Kriener, M. W. Haverkort, T. Lorenz, G. S. Uhrig, L. Jongen, A. Möller, G. Meyer, and M. Grüninger, Phys. Rev. Lett. 95, 097203 (2005).

${ }^{9}$ T. Imai and F. C. Chou, cond-mat/0301425 (unpublished).

${ }^{10}$ G. Caimi, L. Degiorgi, N. N. Kovaleva, P. Lemmens, and F. C.
Chou, Phys. Rev. B 69, 125108 (2004).

${ }^{11}$ A. Krimmel, J. Strempfer, B. Bohnenbuck, B. Keimer, M. Hoinkis, M. Klemm, S. Horn, A. Loidl, M. Sing, R. Claessen and M. von Zimmermann,Phys. Rev. B 73, 172413 (2006).

${ }^{12}$ P. M. de Wolff, T. Janssen, and A. Janner, Acta Crystallogr., Sect. A: Cryst. Phys., Diffr., Theor. Gen. Crystallogr. 37, 625 (1981).

${ }^{13}$ S. van Smaalen, Z. Kristallogr. 219, 681 (2004).

${ }^{14}$ V. Petricek, M. Dusek, and L. Palatinus, The Crystallographic Computing System JANA2000 (Institute of Physics, Praha, Czech Republic, 2000).

${ }^{15}$ T. Sasaki, M. Mizumaki, T. Nagai, T. Asaka, K. Kato, M. Takata, Y. Matsui, and J. Akimitsu, cond-mat/0509358 (unpublished).

${ }^{16}$ H. Z. Cummins, Phys. Rep. 185, 211 (1990).

${ }^{17}$ In monoclinic symmetry, the 479 measured reflections reduce to 148 observed unique main reflections and 97 observed unique satellite reflections. Structure refinements within superspace symmetry $P 2 / n\left(q_{1} q_{2} 0\right) 00$ have resulted in a good fit to the data with $R_{\text {all }}=0.0440, R_{\text {main }}=0.0395$, and $R_{\text {sat }}=0.0972$. Significant deviations of the twin volume ratio from 1:1 could not be found. Structure refinements within orthorhombic superspace symmetry $\operatorname{Pmmn}\left(q_{1} q_{2} 0\right)\left(-q_{1} q_{2} 0\right) 000000$ have resulted in a fit to the data of equal quality, with $R_{\text {all }}=0.0441, R_{\text {main }}=0.0395$, and $R_{\text {sat }}$ $=0.0975$. 